\title{
Time Based Location Management using CACHE Scheme for Mobile Environment
}

\author{
P.Sasikumar ${ }^{1}$, S.Pradeep ${ }^{2}$, K.Gnanathangavelu ${ }^{3}$ \\ 1. Assistant Professor, Computer Science and Engineering, Paavai College of Engineering, Tamil Nadu ,India \\ 2. Assistant Professor, Computer Science and Engineering, MNSK College of Engineering, Tamil Nadu, India \\ 3. Lecturer, Computer Science, Mizan- Tepi University,Ethipoia
}

\begin{abstract}
Location management refers to the problem of updating and searching the current locations of mobile nodes in a wireless network. The location administration basically is performed by using two registers, HLR (Home Location Register) and VLR (Visitor Location Register). The current location information has to be maintained efficiently in location registers for effective call delivery. In the system it is difficult to deliver a call to the users at an optimal time interval. This is used to manage and reduce the level of time of call forwarding from the caller to the callee. To make it efficient, the sum of update/lookup costs of a location database must be minimized. To reduce the cost of location database, this paper we are using the concept of time based location administration system. This paper proposed the CACHE scheme which register the user's frequent movement of repeated visit of same location and maintain the user's detail up-to-date. This optimizes the time-interval which minimize the location administration cortical factors to be established.
\end{abstract}

\section{Motivation}

A major challenge in personal communication service network (mobile network) is the locations updates and call delivery. Location update of mobile stations is mandatory to deliver the calls and data in PCS network. Users will not present in the same place. They will move from one place to other places. So we have to update the current location of the mobile user for call delivery.

\section{Problem statement}

In location registration, the MS move here and there, location registration for call tracking is always needed. These operations cause much network traffic. The location update allows a mobile device to inform the cellular network, whenever it moves from one location area to the next. If there are more VLR in cellular network, the location update increases it include the following steps as follows,

- The $\mathrm{CN}$ request the current location from the HLR and the location is updated. So the CN can send the data MN directly.

- Now the MN change it is location and updated in VLR. In this case the CN does not know about the new location of the MN.

- If the CN continuously sent the data but the MN does not receives the data. Now the CN request the HLR and updates the MN's new location then the data are transfer to MN.

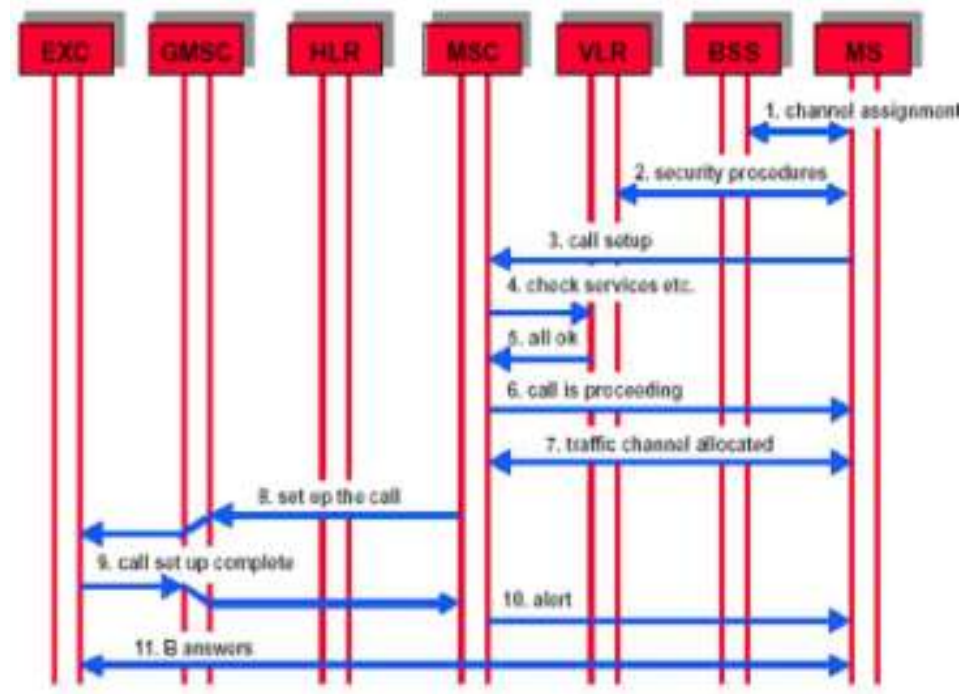

Fig 1. Location Update 


\section{Objectives}

Time based method is a major parameter in the location administration system which is used to reduce the time interval when a caller forwards the call to the callee. The main motive of this project is to forward the call directly to the callee's VLR without sending the call to the HLR. This optimal time interval also minimizes the location administration cost.

\section{Design goals}

- Tunable: Can be tuned to exploit user mobility patterns and uneven regional distribution for efficiency.

- Flexible: Provide greater flexibility in terms of deployment of location information so as to achieve a balance of update and lookup costs.

- Adaptive: Dynamically adapt to a desirable balance by automating tracking of user patterns, and exploit a change in user patterns to the extent of the fluctuation Location administration schemes typically use two extremes,

(i) Up-to-date and exact location information at all sites, requiring huge bandwidth for each location update but minimizing lookup cost; or

(ii) Storing no location information at any site, requiring no update Location administration refers to the problem of updating and searching the current locations of mobile nodes in a wireless network. To make it efficient, the sum of update/lookup costs of a location database must be minimized.

The proposed system consists of time-based update method with paging methodology to get the optimal time-interval for call delivery which minimizes the location administration cost and increases the efficiency. The location administration cost varies according to the number of update made in the location registers. The paging methodology contains paging table for maintaining the dynamic users based on priority. The priority for dynamic users in paging table is given based on the call arrival rates. Based upon the priority the current location information of the dynamic users are updated to the location registers so that efficient call delivery can be achieved at an optimal time interval.

The dynamic mode location administration helps to deliver calls efficiently to the dynamic users using paging methodology. The paging methodology helps to deliver calls to the dynamic users within short time interval based upon their priority. The dynamic users receives frequent calls possess higher priority and the dynamic users receives calls rarely possess low priority in the paging table. The time based update method is used for updating the current location information of dynamic users at regular time interval .Thereby dynamic mobile nodes can be tracked within short interval of time and with minimum location administration cost.

- In no update mode, the user will stay in the fixed location and will not move from location area.

- This scheme considers target tracking of the static users and optimizes time interval when a person calls another person in the static mode.

- This project focuses on optimizing the time interval by directly sending the call to VLR without reaching caller's VLR.

- When users repetitively move between two or more Location Areas, updates are continuously performed unnecessarily. So the control channels may be used to other jobs.

-When the time based method is applied, the optimal time interval minimizes the location administration cost.

- Therefore, it needs to analyze the optimal time interval to save the signaling channels and to forward the call efficiently

\section{Methodology}

Location administration is one of the important issues in personal communications services network. Location administration keeps track of the mobile terminals moving from place to place in PCS network. There are two basic problems such as,

- Call delivery

- Location update

Location administration in mobile communication system is concerned with the cellular network functions necessary to allow the users to be reached wherever they are in the network coverage area.

Cellular networks are spreading rapidly, leading to overloaded systems, unacceptable delays, and increasing computational costs due to inefficient Location Administration (LM). Location administration is a key issue in personal communication service (PCS) networks. Performance analysis plays important roles in the implementation of location administration methods and system design in PCS networks. Existing PCS networks have the home location registers (HLRs) and visitor location registers (VLRs) architecture for location administration. The HLR acts as the primary database repository for subscriber information used to provide control and intelligence, and therefore manage mobile subscriber profiles and location, as well as handling supplementary services. HLR contains static information about the location of the user regardless of their mobility. One of the MSC's maintains a location database called HLR. Home Location Register (HLR) which 
keeps the client profiles, including the real-time locations of mobile clients. In addition, other MSC's maintain a Visitor Location Register (VLR) for the location information of the mobile clients, which are currently within the cell responsible by the MSC. When a mobile client moves out of its current cell and enters into another cell, a new entry of the client location is added into the VLR of the new cell, and then the HRL will be updated accordingly. In locating a mobile client, the VLR of the cell, where the query is initiated, will be searched first. If the client cannot be found in the VLR, a request will be sent to the HLR of the client to find out its location.

A VLR is a database, similar to a HLR, which is used by the mobile network to temporarily hold profiles of roaming users (users outside their home area). This VLR data is based on the user information retrieved from a HLR. MSCs use a VLR to handle roaming users. When a subscriber is visiting a network out his home location range, the corresponding VLR of the visited location queries the HLR for the subscriber information. The VLR also stores the mobile subscriber profile but this information is kept just while the user is roaming within its network range. Each VLR is connected to multiple Mobile Switching Centers (MSCs), which operate in the transport network in order to aid in handoffs and to locate users more easily. To reduce the cost of de-registration, VLR records are not deleted until the database is full. This prevents the unnecessary repetition of the registration process from one coverage area to another. The dynamic mode location administration helps to deliver calls efficiently to the dynamic users using paging methodology. The paging methodology helps to deliver calls to the dynamic users within short time interval based upon their priority. The dynamic users receives frequent calls possess higher priority and the dynamic users receives calls rarely possess low priority in the paging table. The time based update method is used for updating the current location information of dynamic users at regular time interval Thereby dynamic mobile nodes can be tracked within short interval of time and with minimum location administration cost.

\subsection{Static mode with maximum call arrivals}

The user who is in static mode, will not move anywhere from its original place. This will be noted while requesting for a mobile connection. Therefore in HLR the current place of the user will be uploaded. As the details have been updated, HLR is not disturbed at any time. Where the call from the caller is just forwarded to the static VLR of callee. When the user receives more number of calls, then the first call from the caller will be delivered to the callee using paging. Where paging is the method which is used to allow the caller who calls first to the callee in the fixed location. Thus scheme will deliver the call to the callee using priority intelligent and time delay is reduced. Therefore all number of calls is delivered easily to the callee by directly forwarding to the VLR by avoiding the HLR to forward the call. Thus maximum number of call has been forwarded to the callee by avoiding traffic.

\section{2. Static mode with minimum call arrivals}

The user will receive minimum call from the caller. So, much traffic will not occur. In the static mode the user will stay in the location where the callee stays ideal. Therefore, for minimum call receiving the call will be directly forwarded to the callee's VLR from the caller. Here in this we do not need any paging system for prioritizing the call from the caller. Thus paging system is not much been used in this module. As one or less call be forwarded to the callee, paging system is not required. But cache memory will save the caller's current status. And the details will be removed as soon as the user moves to new location. Here the caller will be free to call the callee, as the mode is with minimum call setup. So that traffic has been reduced while forwarding the call from the caller to the callee.

\subsection{Paging system with cache scheme}

The term paging is said to be updating the user's current location in the specified VLR's cache memory. The paging technology is to dedicate the call by giving priority to the FCFS basis. In the paging memory-administration scheme, the VLR rescues data from HLR in same-size blocks called pages. The cache memory scheme is the one used to have the user details until the user move on to the new location. Thus the paging and cache memory is used for priority and manage the user details. A cache is a component that improves by transparently storing data such that future requests for that data can be served faster. The data that is stored within a cache might be values that have been computed earlier or duplicates of original values that are stored elsewhere. If requested data is contained in the cache, this request can be served by simply reading the cache, which is comparably faster.. Hence, the more requests can be served from the cache the better the overall system routine. As opposite to a buffer, this is achieved explicitly by a client, a cache supplies data transparently. This means that a client who is demanding data from a organization is not aware that the cache exists, which is the source of the term cache. To be cost effective and to enable an effective lookup of data, caches are comparably trivial. References exhibit temporal locality if data is requested again that has been recently requested already. 


\subsection{Dynamic mode with maximum call arrivals}

This module deals with the users who are receiving calls frequently while in motion. The mobility of the user will be noted by arising a query for a mobile connection establishment. Since the users are always in motion their current location information's will be updated perdiocally to the location registers namely HLR and VLR. The threshold value given to the dynamic users who are receiving calls frequently will be low so that their location information's will be maintained effectively in the HLR. Therefore all the calls will be delivered easily to the callee within short time interval by directly forwarding it to the HLR without referring the old VLR. Thus maximum number of call has been forwarded to the callee by avoiding traffic.

\section{5. Dynamic mode with minimum call arrivals}

This module deals with the users who are receiving calls rarely while in motion. The mobility of the user will be noted by arising a query for a mobile connection establishment. Since the users are always in motion their current location information will be updated periodically to the location registers namely HLR and VLR. The threshold value given to the dynamic users who are receiving calls rarely will be high so that their location information will be maintained effectively in the HLR. Here the network traffic will not affect the call delivery process since the users will be receiving calls rarely. Therefore all the calls will be delivered easily to the callee within short time interval by directly forwarding it to the HLR without referring the old VLR.

\section{Implementation}

To implement this project, first the cost equations are generated then these equations are used for the comparative study between different sets of values of signaling cost that are generated between different links of the network.

\subsection{Cost Equations}

There are some parameters which are required to implement the proposed system. These parameters are explained in the following given table Cost Variable Parameters. In the first phase, assuming that the signaling cost dominates so for simplicity taking the database access costs as 0 . Some random signaling costs are given in table below. The value of sig 1 takes (cost between the MSC and CACHE) 1 because CACHE is placed as close as MSC. We are comparing the constraints between the different sets for all values of CMR (call-mobility ratio). If plot the cost ratio against CMR where CMR varies from $0-10$. Generally the cost ratio increases with the value of CMR.

TABLE 1 Cost Variable Parameters

\begin{tabular}{|c|c|}
\hline $\begin{array}{c}\text { COST } \\
\text { VARIABLE }\end{array}$ & DESCRIPTION \\
\hline cost_hlr & Update or querying HLR \\
\hline cost_vlr & Update or querying VLR \\
\hline cost_CACHE & Update or querying CACHE \\
\hline sig 1 & $\begin{array}{c}\text { Cost for messaging between } \\
\text { CACHE and MSC }\end{array}$ \\
\hline sig 2 & $\begin{array}{c}\text { Cost for messaging between } \\
\text { CACHE and HLR }\end{array}$ \\
\hline sig 3 & $\begin{array}{c}\text { Cost for messaging between } \\
\text { two CACHE Q Locality of } \\
\text { movement }\end{array}$ \\
\hline
\end{tabular}

When the CMR is low the mobility rate is high so that the registration cost will be high. In this situation the saving cost will be obtained when the MS changes the BLOCK that means, the MS registers with the HLR only when it is crossing one block. When CMR is high, the mobility rate is low the cost saving will be obtained from the call arrival. If we take the value in the following way then the cost ratio according to the system.

In mobile computing environment, how to locate a mobile effectively and how to reduce the cost of the system tracking the mobile, i.e. location administration, is a challenging frontier. The basic location administration scheme of personal communications services (PCSs) networks, such as GSM and IS-41, requires that location updating be initiated immediately whenever a mobile crosses the boundaries of location areas. Obviously, this scheme wastes the system's resources substantially since a lot of location updating may be unnecessary. Based on layered location areas and ring search scheme and a controllable parameter, a new location administration scheme, in which almost no location updating is needed when a mobile crosses 
boundaries of location areas, is proposed to reduce the cost of location administration. Moreover, a probability formula of a mobile residing in each location area layer between two successive call arrivals is proposed by means of constructing a Markov chain and using its equilibrium equation. Below table mention the Comparative Study between Different Sets of Values.

TABLE 2 .Comparative Study between Different Sets of Values

\begin{tabular}{|l|l|}
\hline \multicolumn{1}{|c|}{ COST VARIABLE } & \multicolumn{1}{c|}{ EXPRESSIONS } \\
\hline $\begin{array}{l}\text { x1(Cost for location registration during intra block } \\
\text { movement if sending id of the current MSC to } \\
\text { HLR is required) }\end{array}$ & $\begin{array}{l}\text { 2cost_vlr+cost_CACHE+cost_hlr+6sig1+2sig2(e.g.:1.cost } \\
\text { of newVLR/MSC(cost_vlr)2.cost of old VLR/MSC } \\
\text { (cost_vlr)3.cost of CACHE(cost_CACHE)4.cost of HLR } \\
\text { (cost_hlr). Six messages exchanged between CACHE and } \\
\text { old VLR/new VLR. Two messages between CACHE and } \\
\text { HLR }\end{array}$ \\
\hline $\begin{array}{l}\text { x2 (Same as x1 but when sending id is not } \\
\text { required) }\end{array}$ & 2cost_vlr+cost_CACHE+6sig1 \\
\hline $\begin{array}{l}\text { x3 (Cost for location registration after an inter } \\
\text { block movement) }\end{array}$ & 2cost_vlr+2cost_CACHE+cost_hlr+6(sig1+sig2) \\
\hline $\begin{array}{l}\text { y1(cost for location tracking if remote pointer for } \\
\text { the called MS is available) }\end{array}$ & 2cost_vlr+cost_CACHE+4sig1+2sig3 \\
\hline $\begin{array}{l}\text { y2(Cost for location tracking/call delivery if } \\
\text { remote pointer for the called MS is not available } \\
\text { and HLR query is required for current serving } \\
\text { CACHE) }\end{array}$ & 2cost_vlr+2cost_CACHE+cost_hlr+4(sig1+sig2 \\
\hline $\begin{array}{l}\text { y3 (Same as y2 but here HLR query is required for } \\
\text { current MSC) }\end{array}$ & 2cost_vlr+cost_CACHE+cost_hlr+4(sig1+sig2) \\
\hline $\begin{array}{l}\text { y4(Same as y2 but here HLR query is required for } \\
\text { current }\end{array}$ & 2cost_vlr+cost_CACHE+4sig1 \\
\hline
\end{tabular}

TABLE 4 Cost values

\begin{tabular}{|l|l|l|l|l|}
\hline \multirow{3}{*}{ Cost ratio } & Set 1 & Set 2 & Set 3 & Set 4 \\
\cline { 2 - 5 } & 0.56 & 0.72 & 0.47 & 0.61 \\
\hline
\end{tabular}

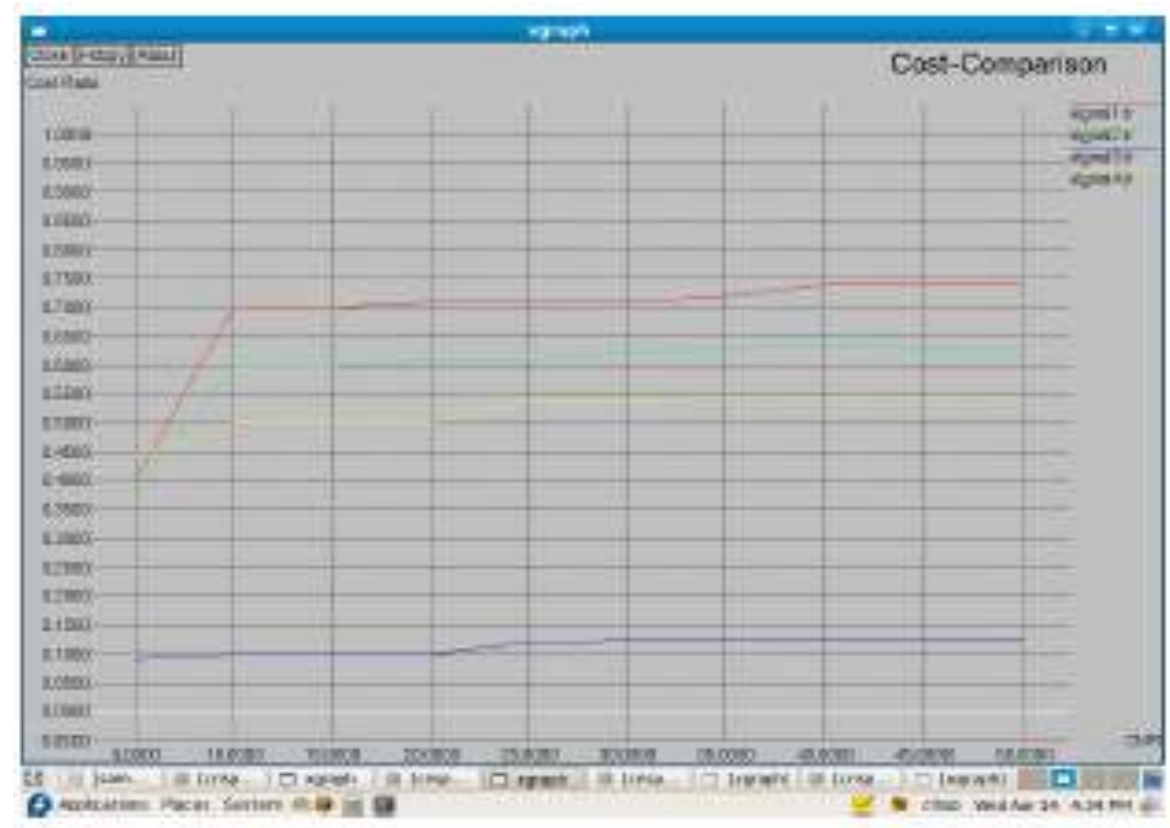

Fig. 2 Comparison graph1 


\begin{tabular}{|}
\begin{tabular}{|c|c|c|c|}
\hline $\begin{array}{c}\text { Set } \\
\mathbf{1}\end{array}$ & $\begin{array}{c}\text { Sig1(MS } \\
\text { C- } \\
\text { CACHE) }\end{array}$ & $\begin{array}{c}\text { Sig2(CACH } \\
\text { E-HLR) }\end{array}$ & $\begin{array}{c}\text { Sig3(CACHECA } \\
\text { CHE) }\end{array}$ \\
\hline 1 & 1 & 5 & 2 \\
\hline 2 & 1 & 5 & 10 \\
\hline 3 & 1 & 10 & 2 \\
\hline 4 & 1 & 10 & 10 \\
\hline
\end{tabular}
\end{tabular}

\begin{tabular}{|l|c|c|c|c|}
\hline \multirow{2}{*}{ Cost ratio } & Set 1 & Set 2 & Set 3 & Set 4 \\
\cline { 2 - 5 } & 0.56 & 0.72 & 0.47 & 0.61 \\
\hline
\end{tabular}

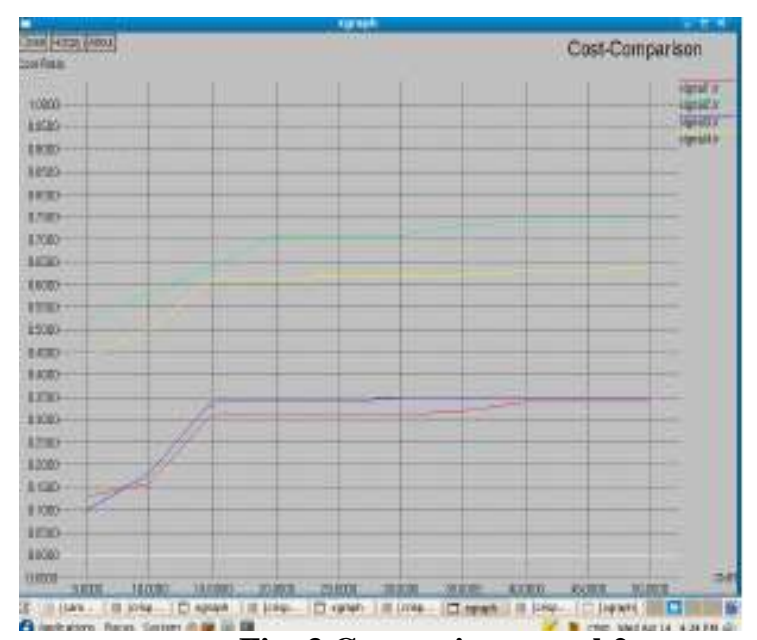

Fig. 3 Comparison graph2

\subsection{Observation from above Tables}

Comparing between set 1 andset 2 demonstrates that sig3 is used during the location tracking/call delivery process (during the high CMR). At this time signaling messages are exchanged between the two CACHE. No signaling messages are sent between the two CACHE during the registration process. Cost ratio decreases under all CMR values in case of set 3 as compared to set 1 because the signaling cost between the CACHE and the HLR (sig2) has increased to reduce the costly HLR access. In this case cost for the standard system will be higher. Now, if the value between the two CACHE is placed according to the table and the value between the CACHE and the HLR have increased then the result will be in the following:

Set 3 gives the better result in set 2 than set 1

Placing the value between the two CACHE as 10 and between the CACHE and the HLR as 50 in set4 Performance improves of set 3 from tables.

Performance_improvement $=((0.48-0.40) /(0.50)) * 100=16.67 \%$

\section{Benefits and limitations}

A new simple location administration by registering representative VLR of group of several VLRs regionally and broadcasting for searching a mobile user, so called rVLR-B. It manages the representative VLR of several VLRs and register mobile users location. When set up the call path between mobile users, the VLR of the caller queries callee's rVLR for searching the location of callee instead of requesting to VLR of callee then rVLR broadcast the callee's location to all VLR of the region concurrently. Location registration is only performed when a mobile user visits a new rVLR network area from current area. Using the rVLR-B, the speed of the call delivery in location administration can be increased. Registration cost of user location is reduced. Movement based location scheme helps to create block which is the group of MSCs so that the registration with HLR will be performed only after crossing the block After training, the blocks are created for particular numbers of MSCs. In standard GSM system the registration is performed only after crossing the location area that is group of cells. After creation of block a block register is maintained to control each block using local and remote pointers. So, the two levels GSM standard system is now three level systems in which a block register is introduced between MSC and HLR. 


\section{Conclusion}

Time based location administration scheme for users has been introduced. The proposed scheme proposes the efficient tracking of the user. This scheme considers target tracking of the static users and calculates the optimal time interval when a person calls another person. When the time based method is applied, the optimal time interval which minimizes the location administration cost can be detected. Using such a scheme helps in minimizing the time consumed in network application and hence to improve the target tracking the optimal time interval to minimize the location cost is dependent on the call arrival rate and user mobility Therefore, it needs to analyze the optimal time interval to save the signaling channels and to forward the call efficiently.

\section{References}

[1] Bjorn Landfeldt, "A Dynamic Location management on Scheme based on individual metrics and coordinates" IEEE Global Telecommunications Conference, Vol. 3, pp. 1426-1430, 2006.

[2] Jie Li, Yi Pan and Yang Xiao, "A Dynamic HLR Location management Scheme for PCS”, IEEE.

[3] Kwok, YK, "A quasi-static cluster-computing approach for dynamic channel assignment in cellular mobile communication systems" IEEE 10.1109/VETECF.1999.797357 1999.

[4] Munadi, R.; Ismail, M.; Abdullah, M.; Misran, N.; Electr. Eng. Dept., Syiah Kuala Univ., Banda Aceh, Indonesia,” Location management cost reduction using fuzzy logic in cellular radio network", Space Science and Communication (IconSpace), 2011 IEEE International Conference.

[5] Networks" IEEE Trans. 0-7803-8356-7/04/\$20.00 IEEE INFOCOM 2004.

[6] Rodriguez-Dagnino, R.M.; Takagi, H.;

Monterrey Inst. of Technol. \& Higher Educ. (ITESM), Monterrey," Movement-Based Location management for General Cell Residence Times in Wireless Networks", IEEE Vehicular Technology Society, 2007.

[7] Travis Keshav "Location management in Wireless Cellular Networks" IEEE Trans. 2005: v.54, no.2 687-708.

[8] Vincent W S. Wong and Victor C. M. Leung, "Location management for Next Generation Personal Communication Networks" Paper accepted by IEEE Network, Special Issue on Next Generation Wireless Broadband Networks.

[9] Yong Lee, "Optimal Time-Interval for Time-based Location Update in Mobile Communications"0-7803-7632-3/02/\$17.000 2002 IEEE.

[10] S. Ratnasamy, B. Karp, L. Yin, F. Yu, D. Estrin, R. Govindan, and S. Shenker. GHT: a geographic hash table for data-centric storage. In ACM Int'l workshop on Wireless Sensor Networks and Applications (WSNA), pages 78-87, 2002.

[11] A. Rowstron and R. Druschel. Pastry: Scalable, Distributed Object Location and Routing for Large-scale Peer-to-peer Systems. Proceedings of the 18th IFIP/ACM Int'l Conf. on Distributed Systems Platform (Middleware 2001), 11:329-350, 2001.

[12] R. Sarkar, X. Zhu, and J. Gao. Double rulings for information brokerage in sensor networks. In ACM Int'l Conf. on Mobile Computing and Networking (MobiCom), pages 286-297, 2006.

[13] I. Stoia, R. Morris, D. Karger, M. F. Kaashoek, and H. Balakrishnan Chord: A Scalable Peer-to-peer Lookup Service for Internet Application. Proceedings of ACM SIGCOMM, 31(4):149-160, 2001

[14] I. Stojmenovic. Home Agent Based Location Update and Destination Search Schemes in Ad Hoc Wireless Networks (a chapter in Advances in Information Science and Soft Computing edited by A. Zemliak and N.E. Mastorakis). WSEAS Press, pages 6-11, 2002.

[15] I. Stojmenovic, A. P. Ruhil, and D. K. Lobiyal. Voronoi diagram and convex hull based geocasting and routing in wireless networks: Research articles. Wireless Communication and Mobile Computing, 6(2):247-258, 2006.

[16] Y.-C. Tseng and T.-Y. Hsieh. Fully power-aware and location-aware protocols for wireless multi-hop ad hoc networks. In Int'l Conf. on Computer Communications and Networks (ICCCN), pages 608-613, 2002.

[17] R. Want, A. Hopper, V. Falco, and J. Gibbons. The active badge location system. ACM Transactions on Information Systems, 10(1):91-102, 1992.

[18] Y. Xue, B. Li, and K. Nahrstedt. A scalable location management scheme in mobile ad-hoc networks. In IEEE Annual Conf. on Local Computer Networks (LCN), pages 102-111, 2001

[19] B. Yang and H. Garcia-Molina. Improving Search in Peer-to-peer Networks. Proceedings of the 22nd Int'l Conf. on Distributed Computing Systems (ICDCS), pages 5-14, 2002.

[20] B. Zhao, J. Kubiatowicz, and A. Joseph. Tapestry: An Infrastructure for Fault-tolerant Wide-area Location and Routing. Tech. Rep. UCB/CSD- 01-1141, University of California at Berkeley, Computer Science Department, 2001. 